\title{
Left Ventricular Diastolic Function Is Closely Associated With Mechanical Function of the Left Atrium in Patients With Paroxysmal Atrial Fibrillation
}

\author{
Jihei Sara Lee, BSc; Chi Young Shim, MD, PhD; Jin Wi, MD; Boyoung Joung, MD, PhD; \\ Jong-Won Ha, MD, PhD; Moon Hyoung Lee, MD, PhD; Hui-Nam Pak, MD, PhD
}

\begin{abstract}
Background: Left ventricular (LV) diastolic dysfunction may be a mechanism of left atrial (LA) electroanatomical remodeling in atrial fibrillation (AF). We evaluated the association between LV diastolic function and LA mechanical function in non-valvular paroxysmal AF (PAF).

Methods and Results: In 286 patients with PAF (males 73\%, 57 \pm 11 years), LA size, indexed LA volume, LV diastolic function, and LA appendage flow velocity (LAA-FV) in sinus rhythm were measured using transthoracic echocardiography, transesophageal echocardiography and cardiac computed tomography. The LA voltage map was obtained using NavX contact mapping. Patients with impaired LA mechanical function (LAA-FV $<58 \mathrm{~cm} / \mathrm{s}, \mathrm{n}=142$ ) showed a higher E/Em ratio (10.3 vs. 9.2, $\mathrm{P}=0.034)$ and lower Em velocity $(6.8 \mathrm{vs} .7 .7 \mathrm{~cm} / \mathrm{s}, \mathrm{P}=0.004)$ than those with preserved function ( $L A A-F V \geq 58 \mathrm{~cm} / \mathrm{s}, \mathrm{n}=144$ ). The patient population displayed weak correlations of $E / E m$ with LAA-FV $(r=-0.19, P=0.003)$ and LA voltage $(r=-0.23, P=0.004)$, but more significant association of $E / E m$ and LAA$F V(r=-0.39, P<0.001)$ for age $\geq 55$ years and $L A$ diameter $\geq 40 \mathrm{~mm}$. E/Em was an independent predictor of $L A A$ mechanical function $(\beta=-0.20, P=0.013)$ even after age, sex, LA size and comorbidities were controlled for.
\end{abstract}

Conclusions: In patients with non-valvular PAF, LA mechanical function is closely related to the degree of LA remodeling and LV diastolic function. Impaired LV diastolic function significantly contributes to LA electoanatomical remodeling in older patients with a larger LA. (Circ $J$ 2013; 77: 697-704)

Key Words: Atrial fibrillation; Diastolic function; Electroanatomical remodeling; Left atrium; Left ventricle

A trial remodeling, both structural and electrical, is a pathophysiological phenomenon observed in atrial fibrillation (AF). ${ }^{1}$ Over the years, various studies have shown that fibrillatory electrical activation causes progressive structural and electrical remodeling of the atria.,3 Subsequently, remodeling impairs the function of the rest of the heart, and this partly explains why this arrhythmia is a major cause of population morbidity and mortality. ${ }^{4,5}$ The reverse seems to be also true: impaired function of the heart, especially that of the ventricles, debilitates the atria, because the left atrium (LA) and the left ventricle (LV) are intricately coupled. For instance, the LA during diastole is under the direct influence of pressure in the LV through the open mitral valve. ${ }^{6}$ Hence, it is not without reason to suspect that the function of the LV, or the lack thereof, would alter the LA in structure and function. Sure enough, many studies to date have come to the conclusion that the state of the LA reflects the diastolic function of the LV. ${ }^{6-9}$ In this context, LV diastolic dysfunction would be a major contributor to LA electroanatomical remodeling observed in paroxysmal AF (PAF). However, no studies so far have conclusively proved that the relationship between the LA and the LV exists in this arrhythmia. Employing a variety of diagnostic tests to obtain data on heart structure, function and electrophysiology of patients undergoing radiofrequency catheter ablation (RFCA), we hypothesized that electroanatomical remodeling of the LA is intimately connected to LV diastolic dysfunction in PAF. Hence, the aim of this study was to evaluate the association between LV diastolic function and LA mechanical function, and to characterize those individuals, in whom LV diastolic function significantly altered LA mechanical function.

\section{Methods}

\section{Patient Selection}

The study protocol adhered to the Declaration of Helsinki and was approved by the Institutional Review Board of Severance Hospital, Yonsei University. All patients included in the study

Received August 9, 2012; revised manuscript received September 16, 2012; accepted October 23, 2012; released online November 30,

2012 Time for primary review: 11 days

Yonsei University Health System, Seoul, Republic of Korea

Mailing address: Hui-Nam Pak, MD, PhD, and Chi Young Shim, MD, PhD, Yonsei University Health System, 250 Seungsanno,

Seodaemun-gu, Seoul 120-752, Republic of Korea. E-mail: hnpak@yuhs.ac; cysprs@yuhs.ac

ISSN-1346-9843 doi:10.1253/circj.CJ-12-1009

All rights are reserved to the Japanese Circulation Society. For permissions, please e-mail: cj@j-circ.or.jp 


\begin{tabular}{|c|c|c|c|}
\hline & LAA-FV $<58 \mathrm{~cm} / \mathrm{s}(\mathrm{n}=142)$ & LAA-FV $\geq 58 \mathrm{~cm} / \mathrm{s} \quad(\mathrm{n}=144)$ & P value $^{\star}$ \\
\hline Age, years & $58.5 \pm 10.3$ & $55.2 \pm 12.0$ & 0.012 \\
\hline Age >75, n (\%) & $7(4.9)$ & $8(5.6)$ & 0.812 \\
\hline Male sex, n (\%) & $98(69.0)$ & $109(75.7)$ & 0.206 \\
\hline $\mathrm{BMI}, \mathrm{kg} / \mathrm{m}^{2}$ & $24.5 \pm 2.9$ & $24.8 \pm 3.0$ & 0.493 \\
\hline Hypertension, n (\%) & $72(50.7)$ & $65(45.1)$ & 0.346 \\
\hline Diabetes mellitus, $\mathrm{n}(\%)$ & $22(15.5)$ & $13(9.0)$ & 0.095 \\
\hline Heart failure, $n(\%)$ & $10(7.0)$ & $5(2.8)$ & 0.092 \\
\hline Stroke, n (\%) & $19(13.4)$ & $7(4.9)$ & 0.012 \\
\hline TIA, n (\%) & $2(1.4)$ & $1(0.7)$ & 0.512 \\
\hline Stroke + TIA, n (\%) & $21(14.8)$ & $8(5.6)$ & 0.018 \\
\hline $\mathrm{CHADS}_{2}$ Score & $1.08 \pm 1.19$ & $0.74 \pm 0.90$ & 0.008 \\
\hline
\end{tabular}

${ }^{*} \mathrm{P}<0.05$ was considered statistically significant.

BMI, body mass index; LAA-FV, left atrial appendage flow velocity; PAF, paroxysmal atrial fibrillation; TIA, transient ischemic attack.

provided proper written informed consent. The study enrolled 286 patients (73\% males, $57 \pm 11$ years) who underwent RFCA for symptomatic drug-refractory PAF. The study's exclusion criteria were as follows: (1) persistent or permanent AF; (2) $\mathrm{AF}$ at the time of transesophageal echocardiography (TEE); (3) LA anterior-posterior diameter $>55 \mathrm{~mm}$ measured by transthoracic echocardiography (TTE); (4) the presence of intracardiac thrombi; (5) AF with significant valvular disease or structural heart disease; (6) previous AF ablation; and (7) history of cardiac surgery. All subjects underwent TEE, TTE, and cardiac computed tomography (CT) in preparation for the ablation procedure. All antiarrhythmic drugs were discontinued 5.5 lives prior to RFCA. A total of 286 patients were categorized into 2 groups based on their left atrial appendage (LAA) flow velocity (FV) measured by TEE. It should be noted that the values of LAA-FV were taken only when the patients were confirmed to be in sinus rhythm. Those whose LAA-FV was $<58 \mathrm{~cm} / \mathrm{s}$ were classified into the low LAA-FV group while those whose LAA-FV $\geq 58 \mathrm{~cm} / \mathrm{s}$ were classified into the high LAA-FV group. This particular value of $58 \mathrm{~cm} / \mathrm{s}$ was the median LAA-FV among the 286 patients included in the study.

\section{Echocardiographic and CT Evaluation of the Heart}

TTE was conducted using commercially available devices (Sonos 5500, Philips Medical System, Andover, MA, USA or Vivid 7, GE Vingmed Ultrasound, Horten, Norway), and standard M-mode, 2D and Doppler images were acquired in the parasternal and apical views. Standard 2D measurements were obtained in the left lateral position as recommended by the American Society of Echocardiography..$^{10} \mathrm{LV}$ mass was calculated using the recommended formula, and the mass was further divided by body surface area (BSA) to produce the LV mass index. ${ }^{11}$ In addition, using the pulse wave Doppler method, the mitral inflow peak velocity (E) was measured by placing a sample volume at the opening level of the mitral valve leaflet tips while the tissue Doppler-derived diastolic mitral annular velocity (Em) was measured from the septal corner of the mitral annulus in the apical 4-chamber view. TEE was used to measure the Doppler pulmonary vein (PV) FV, as well as the LAA$\mathrm{FV}$, in all patients. Among the LAA Doppler FVs, we measured the LAA emptying FV as a parameter of LA function. At least 3 consecutive beats were measured and averaged for Doppler-derived parameters.

Furthermore, in order to define the anatomic structure of the LA and PVs of the patients in detail, 3D spiral CT (64
Channel, Light Speed Volume Ct, Philips, Brilliance 63, The Netherlands) was performed in all patients. The 3D spiral CT images of the LA were analyzed on an imaging processing workstation (Aquarius, Terarecon, Inc, USA). Each LA image was divided into portions according to its embryological origin as follows: the venous LA (posterior LA including the antrum and posterior wall), anterior LA (excluding LAA and venous LA), and LAA as previously described. ${ }^{12}$ Of note, the regional volume of the LA measured with $\mathrm{CT}$ was divided by the BSA of the respective patient to correct for the influence of body size on volume.

\section{Electrophysiological Mapping}

Intracardiac electrograms were recorded using the Prucka Cardio $\mathrm{Lab}^{\mathrm{TM}}$ electrophysiology system (General Electric Medical Systems, Milwaukee, WI, USA). A 3D electroanatomical map (NavX, St. Jude Medical, Minnetonka, MN, USA) was generated by merging the NavX system-generated 3D geometry of the LA and PVs with the corresponding 3D spiral CT images. This map was then used to guide the RFCA procedure. A decapolar catheter (Bard Electrophysiology, Lowell, MA, USA) and a duo-decapolar catheter (St. Jude Medical) were inserted into the left femoral vein to map the high right atrium (RA), low RA, and the coronary sinus, and a quadripolar catheter was advanced and placed in the superior vena cava. To gain access to the LA, a double trans-septal puncture approach was taken, and multiview pulmonary venograms were obtained. Thereafter, using a long sheath (Schwartz left 1, St. Jude Medical), a circumferential PV mapping catheter (Lasso; Biosense-Webster, Diamond Bar, CA, USA) was also inserted. Intravenous heparin was injected in order to have systemic anticoagulation with an activated clotting time of 350-400 s. Using a multipolar ring catheter (Lasso, Johnson \& Johnson, Diamond Bar, CA, USA), a 3D LA voltage map was generated from contact bipolar electrograms of 350-400 points on the LA endocardium during high RA pacing (pacing cycle length $500 \mathrm{~ms}$ ). The bipolar electrograms were filtered at $32-300 \mathrm{~Hz}$. Color-coded voltage maps were generated by recording bipolar electrograms and measuring peak-to-peak voltage as previously described. ${ }^{12}$ However, when frequently re-initiating AF required more than 3 electrical cardioversions, the LA voltage map was not constructed. In the end, 171 of the 286 patients had a complete set of LA and LAA voltage data. For further comparisons, these 171 patients were divided again according to LA voltage and echocardiographic measurement 


\begin{tabular}{|c|c|c|c|}
\hline & LAA-FV $<58 \mathrm{~cm} / \mathrm{s}(\mathrm{n}=142)$ & LAA-FV $\geq 58 \mathrm{~cm} / \mathrm{s}(\mathrm{n}=144)$ & P value* \\
\hline \multicolumn{4}{|c|}{ TTE: 2D and Doppler parameters } \\
\hline LA diameter, mm & $40.7 \pm 5.8$ & $39.4 \pm 5.5$ & 0.040 \\
\hline LVEDD, mm & $49.5 \pm 4.5$ & $49.5 \pm 4.0$ & 0.881 \\
\hline LVESD, mm & $32.2 \pm 4.7$ & $32.3 \pm 4.1$ & 0.836 \\
\hline LVEF, \% & $62.3 \pm 9.0$ & $63.4 \pm 8.0$ & 0.267 \\
\hline LV mass index, $\mathrm{g} / \mathrm{m}^{2}$ & $97.0 \pm 18.7$ & $95.2 \pm 20.3$ & 0.451 \\
\hline E velocity, $\mathrm{cm} / \mathrm{s}$ & $67.4 \pm 20.4$ & $68.5 \pm 21.6$ & 0.710 \\
\hline Em velocity, $\mathrm{cm} / \mathrm{s}$ & $6.8 \pm 2.2$ & $7.7 \pm 2.3$ & 0.004 \\
\hline $\mathrm{E} / \mathrm{Em}$ & $10.3 \pm 4.4$ & $9.2 \pm 3.6$ & 0.034 \\
\hline \multicolumn{4}{|l|}{ TEE: Doppler parameters } \\
\hline LAA-FV, cm/s & $40.8 \pm 11.8$ & $75.1 \pm 13.5$ & $<0.001$ \\
\hline \multicolumn{4}{|l|}{ Right PV } \\
\hline Systolic FV cm/s & $41.3 \pm 17.2$ & $50.1 \pm 22.5$ & 0.001 \\
\hline Diastolic FV, cm/s & $50.0 \pm 17.8$ & $46.6 \pm 13.8$ & 0.064 \\
\hline Systolic/diastolic ratio & $0.89 \pm 0.43$ & $1.12 \pm 0.47$ & $<0.001$ \\
\hline \multicolumn{4}{|l|}{ Left PV } \\
\hline Systolic FV, cm/s & $48.7 \pm 18.4$ & $55.5 \pm 16.8$ & 0.004 \\
\hline Diastolic FV, cm/s & $46.7 \pm 18.7$ & $41.0 \pm 13.2$ & 0.008 \\
\hline Systolic/diastolic ratio & $1.15 \pm 0.52$ & $1.43 \pm 0.48$ & $<0.001$ \\
\hline \multicolumn{4}{|l|}{ CT: 3D indexed volume } \\
\hline $\mathrm{LA}, \mathrm{ml} / \mathrm{m}^{2}$ & $65.0 \pm 18.0$ & $59.6 \pm 18.2$ & 0.013 \\
\hline $\mathrm{LAA}, \mathrm{ml} / \mathrm{m}^{2}$ & $5.6 \pm 2.5$ & $4.9 \pm 2.4$ & 0.017 \\
\hline \multicolumn{4}{|l|}{ LA voltage } \\
\hline Mean LA, mV & $1.38 \pm 0.63$ & $1.55 \pm 0.64$ & 0.047 \\
\hline $\mathrm{LAA}, \mathrm{mV}$ & $2.54 \pm 1.46$ & $3.17 \pm 1.43$ & 0.003 \\
\hline
\end{tabular}

${ }^{*} \mathrm{P}<0.05$ was considered statistically significant.

$\mathrm{CT}$, computed tomography; E, mitral inflow early diastolic; Em, mitral annulus early diastolic; FV, flow velocity; LA, left atrium; LAA, left atrial appendage; LV, left ventricle; LVEDD, left ventricular end-diastolic dimension; LVESD, left ventricular end-systolic dimension; LVEF, left ventricular ejection fraction; PV, pulmonic vein; TEE, transesophageal echocardiography; TTE, transthoracic echocardiography.

of LA size into 4 groups: (1) small LA with high voltage $(n=46)$, (2) small LA with low voltage $(n=45)$, (3) large LA with high voltage $(n=40)$, and (4) large LA with low voltage $(n=40)$. Those whose LA diameter was $<40 \mathrm{~mm}$ were categorized into the small LA group, and those whose LA voltage was less than the median value were classified as displaying low voltage in their respective groups.

\section{Statistical Analysis}

Continuous variables are presented as the mean \pm standard deviation and categorical variables as absolute and relative frequencies (\%). Among the data, continuous variables were compared between groups with Student's t-test (for 2-group comparisons) and analysis of variance (ANOVA) (for 4-group comparisons) whereas the categorical variables were compared by chi-square test or ANOVA. Simple correlation analyses were performed to test the association of $\mathrm{E} / \mathrm{Em}$ with parameters that reflect LA mechanical function, and those variables that were found to be significant $(\mathrm{P}<0.10)$ from simple linear regression analysis were then included in the multiple linear regression analyses. Here, LAA-FV was assigned as the dependent variable. A P-value $<0.05$ was regarded as statistically significant.

\section{Results}

LA Mechanical Function and Electoanatomical Remodeling Based on the LAA-FV obtained during sinus rhythm, the patients were divided into low LAA-FV (LAA-FV $<58 \mathrm{~cm} / \mathrm{s}$, $\mathrm{n}=142$ ) and high LAA-FV (LAA-FV $\geq 58 \mathrm{~cm} / \mathrm{s}, \mathrm{n}=144$ ) groups; the median LAA-FV of $58 \mathrm{~cm} / \mathrm{s}$ marks the point of separation between these 2 groups (Table 1). Compared with the high LAA-FV group, the patients with a low LAA-FV were significantly older $(\mathrm{P}=0.012)$, and showed a higher prevalence $(\mathrm{P}=0.012)$ and risk of stroke, estimated by the CHADS 2 score $(\mathrm{P}=0.008)$. The parameters reflecting electroanatomical remodeling of the LA and invasive hemodynamic data are summarized in Table 2. Upon close analysis, the LA anterior-posterior diameter was found to be significantly greater among patients with a low LAA-FV ( $\mathrm{P}=0.040)$, signifying more extensive LA structural remodeling for patients with reduced LA function (Figure 1). In order to verify this finding, 3D spiral CT was performed to measure the volumes of the LAA and LA. Indeed, after having been corrected for the influence of $\mathrm{BSA}$, the indexed LA volume $(\mathrm{P}=0.013)$ and LAA volume $(\mathrm{P}=0.017)$ were significantly greater among the patients with a diminished LAA-FV. As expected, the endocardial voltage measured in these areas revealed that the mean LA voltage $(\mathrm{P}=0.047)$ and LAA voltage $(\mathrm{P}=0.003)$ were lower in patients with a reduced LAA-FV when compared with those with a high LAA-FV (Figure 1). In other words, a functionally abated LA tended to be electrically remodeled as well.

\section{Association of LA Mechanical Function and LV Diastolic Function}

To gain insight into the association between the LA and the $\mathrm{LV}$ in our patient population, the LV diastolic parameters were 
A High LA Volume, Low LA Voltage, \& Low LAA-FV

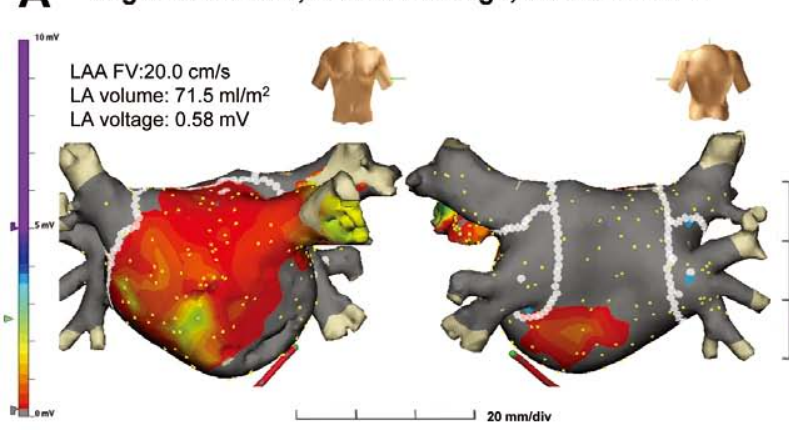

C

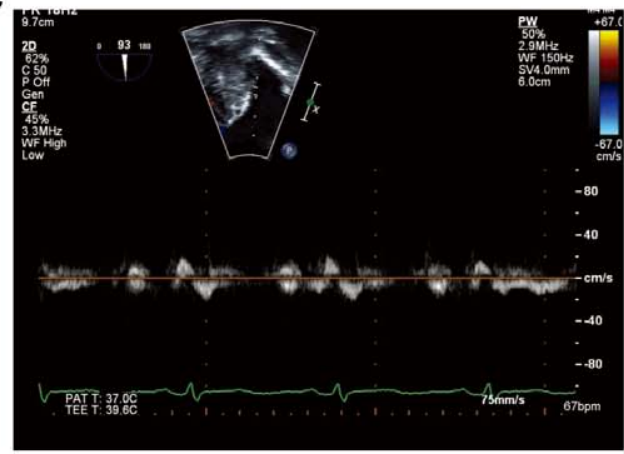

B Low LA Volume, High LA Voltage, \& High LAA-FV

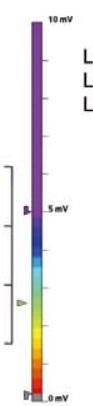

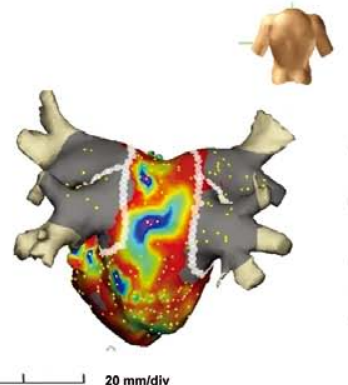

trang

D

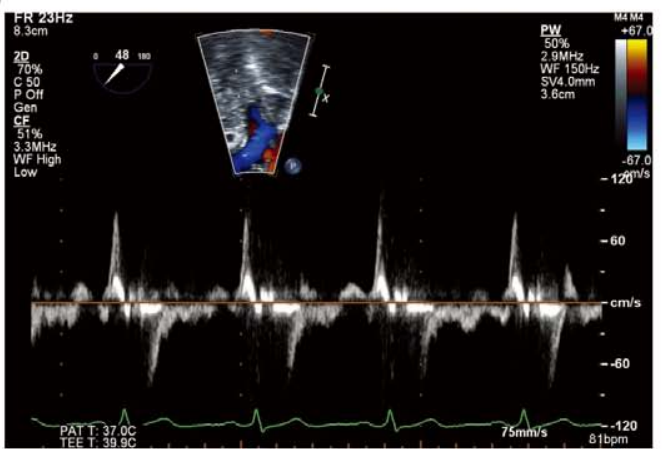

Figure 1. Comparisons of LAA-FV measured by TEE during sinus rhythm and 3D CT-merged electroanatomical voltage maps. (A) CT-merged NavX voltage map of electroanatomically remodeled LA. Gray color shows the endocardial scar zone with voltage $<0.2 \mathrm{mV}$, and the red color shows endocardial voltage $0.2-0.5 \mathrm{mV}$. White circles represent circumferential pulmonary vein ablation lesions. (B) Representative example of minimal LA remodeling with low LA volume and high LA voltage. Green and purple colors on the color-coded 3D electroanatomical voltage map show endocardial voltage 2.0-3.0 mV and >5 mV, respectively. (C,D) Doppler flow velocities in the LAA during sinus rhythm in patient $A(C)$ and patient $B(D)$. CT, computed tomography; FV, flow velocity; LA, left atrium; LAA, left atrial appendage; TEE, transesophageal echocardiography.

compared between the 2 groups. The patients with a low LAA-FV showed a significantly lower Em velocity $(6.8 \pm 2.2$ vs. $7.7 \pm 2.3 \mathrm{~cm} / \mathrm{s}, \mathrm{P}=0.004)$ and a higher $\mathrm{E} / \mathrm{Em}(10.3 \pm 4.4 \mathrm{vs}$. 9.2 $\pm 3.6, \mathrm{P}=0.034$ ) than those with a high LAA-FV (Table 2). There also existed intriguing changes in a Doppler parameter of TEE: patients with a low LAA-FV revealed a diastolic dominant pattern in both pulmonic vein flow velocities. Therefore, in low LAA-FV patients, LV relaxation was more impaired and LV filling pressure was more elevated. When a simple correlation analysis was done, $\mathrm{E} / \mathrm{Em}$ was found to be linearly correlated with LAA-FV $(\mathrm{r}=-0.19, \mathrm{P}=0.003)$ and mean LA voltage $(\mathrm{r}=-0.23, \mathrm{P}=0.004)$. These associations were even stronger for subjects who were $\geq 55$ years of age and had a large LA diameter $(\geq 40 \mathrm{~mm})$ (LAA-FV $\mathrm{r}=-0.39, \mathrm{P}<0.001$; mean LA voltage $r=-0.31, P=0.030$; Figure 2). Subsequent multiple regression analyses found that $\mathrm{E} / \mathrm{Em}$ was an independent predictor of LAA mechanical function $(\beta=-0.20$, $\mathrm{P}=0.013$ ) even after age, sex, indexed LA volume, LV mass index, LV ejection fraction and comorbidities were controlled for (Table 3).

\section{Diastolic Function and LA Electroanatomical Remodeling}

Of the 286 patients, high-density LA voltage mapping data were available for 171 . To assess in detail the changes in various parameters in relation to the extent of LA electroana- tomical remodeling, these 171 patients were categorized into 4 groups, using the LA anterior-posterior diameter measured with echocardiography and the LA voltage. Those whose LA diameter was $<40 \mathrm{~mm}$ were categorized into the small LA group, and $\geq 40 \mathrm{~mm}$ was classified as large LA group. Those whose LA voltage was less than the median voltage value of the small $(1.41 \mathrm{mV})$ and large LA groups $(1.45 \mathrm{mV})$ were classified as displaying low voltage in their respective groups.

These 4 groups were: (1) small LA/high voltage, (2) small LA/low voltage, (3) large LA/high voltage, and (4) large LA/ low voltage (Table 4). The small LA/high voltage group represented the least remodeled stage and the large LA/low voltage group depicted the most extensively remodeled LA (4 group differences in ANOVA: $\mathrm{P}<0.001$ for LA size, $\mathrm{P}=0.001$ for LA volume index, $\mathrm{P}<0.001$ for LA voltage; Table 4). What is striking about the comparisons of these 4 groups is that as remodeling of the chamber progressed, variables known to indicate LA mechanical function and LV diastolic function also changed accordingly. LAA-FV decreased $(\mathrm{P}=0.003)$, and E/Em increased dramatically $(\mathrm{P}<0.001)$ as remodeling progressed (Figure 3). The same can be said for 2 other indicators of diastolic function: left pulmonic vein systolic/diastolic ratio $(\mathrm{P}=0.007)$ and LA diastolic pressure $(\mathrm{P}=0.001)$. 


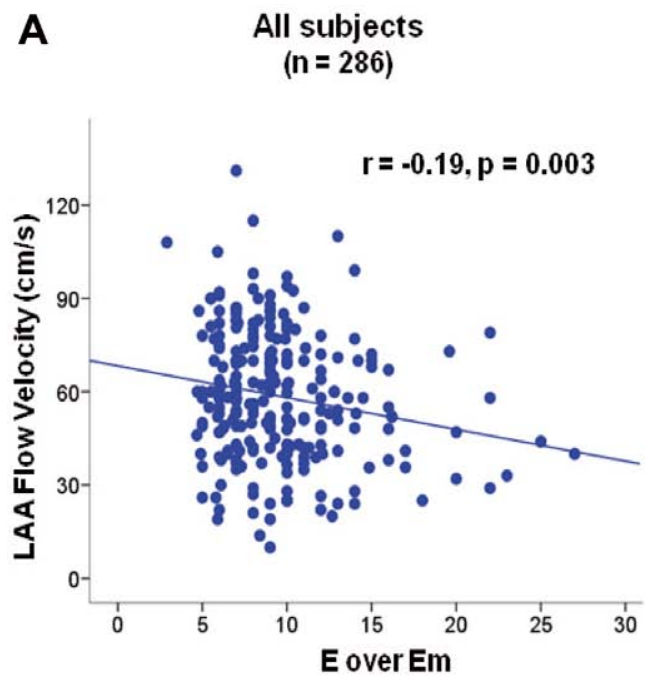

B Age $\geq 55$ years, LA diameter $\geq 40 \mathrm{~mm}$ ( $n=91)$

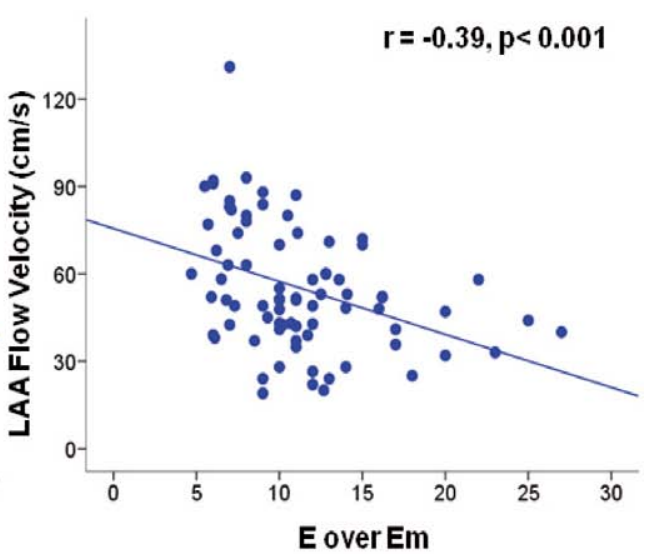

Figure 2. Relationship between left atrial mechanical function and left ventricular diastolic function in all included patients (A) and selected patients aged $\geq 55$ years and left atrial (LA) diameter $\geq 40 \mathrm{~mm}$ (B).

\section{Discussion}

Our results shed light on the direct relationship between the mechanical function of the LA, LA electroanatomical remodeling, and LV diastolic function in PAF patients. Hence, we propose that in PAF patients, LV diastolic dysfunction is an independent predictor of LA mechanical function, which is itself closely associated with the degree of LA electroanatomical remodeling and events or risk of ischemic stroke. Moreover, 4 groups representing 4 progressive stages of electroanatomical remodeling of the LA showed drastic changes in $\mathrm{LV}$ diastolic function when evaluated using echocardiographic parameters or invasively measured LA pressure. In this regard, our study is, to the best of our knowledge, the first attempt to examine electroanatomical remodeling of the LA in patients with PAF through comprehensive interpretation of echocardiographic findings in combination with CT data and intracardiac electrograms.

\section{LAA-FV and Electroanatomical Remodeling of the LA in AF}

Various studies have proven LAA-FV to be a valuable means by which to predict the structure and function of the LA in fibrillation. More specifically, they have argued that larger LA and LAA sizes are associated with lower LAA-FV. ${ }^{13,14}$ What is more, as the contractility of the LA and LAA decreases, indicated by a low LAA-FV, blood stasis becomes a more common occurrence, and the risk of thrombosis and stroke increases. ${ }^{13-16}$ In addition to the mechanical function of the LAA, the degree of electroanatomical remodeling of the LA, as indicated by an increase in LA volume and a decrease in endocardial voltage, was found to be significantly associated with the risk or event of stroke in our previous study and in another study. ${ }^{17-20}$ In this study, we firstly defined the relationship between LA mechanical dysfunction as represented by a low LAA-FV on TEE and global electroanatomical remodeling of the LA in PAF patients through comprehensive interpretation of $\mathrm{CT}^{12}$ and intracardiac electrograms. ${ }^{21}$ Secondly, we found that the risk of stroke estimated by $\mathrm{CHADS}_{2}$ score

\begin{tabular}{|lrcc|}
\hline $\begin{array}{l}\text { Table 3. E/Em as an Independent Predictor of Left Atrial } \\
\text { Mechanical Function }\end{array}$ & $\boldsymbol{\beta}$ & $\mathbf{t}$ & $\mathbf{P}$ value \\
LAA-FV, $\mathbf{c m} / \mathbf{s}(\mathbf{R = 0 . 2 8 6 )}$ & & & \\
Age & -0.01 & -0.13 & 0.899 \\
Male sex & 0.02 & 0.32 & 0.751 \\
BMl & 0.02 & 0.23 & 0.821 \\
Hypertension & 0.05 & 0.66 & 0.511 \\
Diabetes mellitus & -0.07 & -0.98 & 0.331 \\
LVEF & 0.11 & 1.48 & 0.141 \\
LV mass index & 0.11 & 1.49 & 0.139 \\
LA volume index & -0.16 & -2.13 & 0.034 \\
E/Em & -0.20 & -2.50 & 0.013 \\
\hline
\end{tabular}

Abbreviations as in Tables 1,2.

and the prevalence of ischemic stroke or transient ischemic attacks were significantly higher in patients with a low LAAFV.

\section{Relationship Between LA Mechanical Function and LV Diastolic Function}

LA and LV function are known to be interdependent. ${ }^{6-8,22}$ During the LV systolic phase, the LA works as a reservoir and its functionality can be evaluated by the right ventricular systolic pressure and the relaxation properties of the LA. During the early diastolic phase and diastasis, the LA acts as a conduit, and this aspect is mainly affected by LV diastolic function and pressure. Finally, in late LV diastole, the LA works as a pump, whereby LV compliance, pressure, and LA contractility modulate its effectiveness. Thus, we can speculate that LA function in PAF, as estimated by LAA-FV, may be altered by both LV diastolic function ${ }^{23,24}$ and LA myocardial function proper, ${ }^{25}$ which is associated with the degree of electroanatomical remodeling. However, some studies have put 
Table 4. Comparisons of Clinical, Electromechanical and Hemodynamic Characteristics of Patients Based on Size and Endocardial Voltage of the LA

\begin{tabular}{|c|c|c|c|c|c|}
\hline & & & & & \\
\hline & $\begin{array}{l}\text { High voltage } \\
(n=46)\end{array}$ & $\begin{array}{l}\text { Low voltage } \\
(n=45)\end{array}$ & $\begin{array}{l}\text { High voltage } \\
(n=40)\end{array}$ & $\begin{array}{l}\text { Low voltage } \\
(n=40)\end{array}$ & $P$ value \\
\hline Age, years & $53.5 \pm 10.3$ & $57.9 \pm 13.1$ & $57.7 \pm 10.3$ & $60.4 \pm 10.6^{*}$ & 0.079 \\
\hline Male sex, n (\%) & $36(78.3)$ & $27(60.0)$ & $38(95.0)$ & $22(55.0)$ & $<0.001$ \\
\hline CHADS $_{2}$ Score & $0.67 \pm 0.87$ & $0.98 \pm 1.10$ & $1.10 \pm 1.22^{*}$ & $1.30 \pm 1.29^{*}$ & 0.085 \\
\hline TTE: 2D and Doppler $\mathrm{F}$ & & & & & \\
\hline LA size, mm & $35.8 \pm 3.8$ & $36.2 \pm 3.3$ & $44.3 \pm 2.9^{*}, \dagger$ & $45.4 \pm 4.4^{*}, \dagger$ & $<0.001$ \\
\hline LVEF, \% & $64.9 \pm 7.6$ & $64.8 \pm 7.4$ & $64.1 \pm 6.1$ & $64.6 \pm 10.4$ & 0.965 \\
\hline LV mass index, $\mathrm{g} / \mathrm{m}^{2}$ & $96.3 \pm 19.3$ & $90.3 \pm 16.3$ & $97.5 \pm 18.8$ & $107.9 \pm 19.4^{*}$ & $<0.001$ \\
\hline E velocity, $\mathrm{cm} / \mathrm{s}$ & $61.3 \pm 17.4$ & $69.1 \pm 21.3$ & $68.2 \pm 17.5$ & $74.2 \pm 20.5^{\star}$ & 0.037 \\
\hline Em velocity, $\mathrm{cm} / \mathrm{s}$ & $7.3 \pm 2.3$ & $8.0 \pm 2.6$ & $7.1 \pm 1.9$ & $6.4 \pm 2.4^{\dagger}$ & 0.020 \\
\hline $\mathrm{E} / \mathrm{Em}$ & $8.7 \pm 2.6$ & $9.7 \pm 4.0$ & $10.0 \pm 3.2^{*}$ & $12.8 \pm 5.6^{\star}, t, \neq$ & $<0.001$ \\
\hline TEE: Doppler paramet & & & & & \\
\hline LAA-FV & $61.4 \pm 23.1$ & $61.4 \pm 16.2$ & $60.1 \pm 21.3$ & $47.2 \pm 20.0^{\star}, t, \neq$ & 0.003 \\
\hline Right PV S/D ratio & $1.23 \pm 0.39$ & $0.97 \pm 0.49$ & $0.94 \pm 0.44$ & $0.91 \pm 0.48^{*, \dagger}$ & 0.015 \\
\hline Left PV S/D ratio & $1.49 \pm 0.49$ & $1.29 \pm 0.54$ & $1.27 \pm 0.4$ & $1.06 \pm 0.49^{*}, \dagger$ & 0.007 \\
\hline CT: 3D indexed volum & & & & & \\
\hline $\mathrm{LA}, \mathrm{ml} / \mathrm{m}^{2}$ & $56.7 \pm 16.2$ & $57.5 \pm 19.1$ & $65.4 \pm 14.2^{*}$ & $71.5 \pm 17.0^{*}$ & 0.001 \\
\hline $\mathrm{LAA}, \mathrm{ml} / \mathrm{m}^{2}$ & $5.0 \pm 2.6$ & $4.9 \pm 2.3$ & $5.1 \pm 2.3$ & $5.4 \pm 2.4$ & 0.855 \\
\hline LA pressure & & & & & \\
\hline Systolic, mmHg & $21.3 \pm 7.7$ & $20.3 \pm 7.1$ & $20.5 \pm 8.2$ & $24.5 \pm 8.1$ & 0.489 \\
\hline Diastolic, $\mathrm{mmHg}$ & $2.5 \pm 3.7$ & $4.4 \pm 3.1$ & $5.1 \pm 3.9$ & $9.2 \pm 5.4^{\star, t, \neq}$ & 0.001 \\
\hline Mean, $\mathrm{mmHg}$ & $11.0 \pm 4.6$ & $11.9 \pm 4.7$ & $11.8 \pm 6.0$ & $15.5 \pm 7.5^{\star}$ & 0.178 \\
\hline Pacing voltage & & & & & \\
\hline $\mathrm{LA}, \mathrm{mV}$ & $2.01 \pm 0.53$ & $0.98 \pm 0.29^{*}$ & $1.91 \pm 0.4^{\dagger}$ & $0.94 \pm 0.31^{*, \neq}$ & $<0.001$ \\
\hline $\mathrm{LAA}, \mathrm{mV}$ & $3.56 \pm 1.49$ & $2.16 \pm 1.04^{*}$ & $3.91 \pm 1.16^{*, \dagger}$ & $1.79 \pm 0.95^{\star}, \ddagger$ & $<0.001$ \\
\hline
\end{tabular}

${ }^{*} \mathrm{P}<0.05$, compared with small LA/high voltage; ${ }^{\mathrm{P}} \mathrm{P}<0.05$, compared with small LA/low voltage; $\mathrm{P} P<0.05$, compared with large $\mathrm{LA} /$ high voltage. $\mathrm{S} / \mathrm{D}$, systolic/diastolic. Other abbreviations as in Table 2.
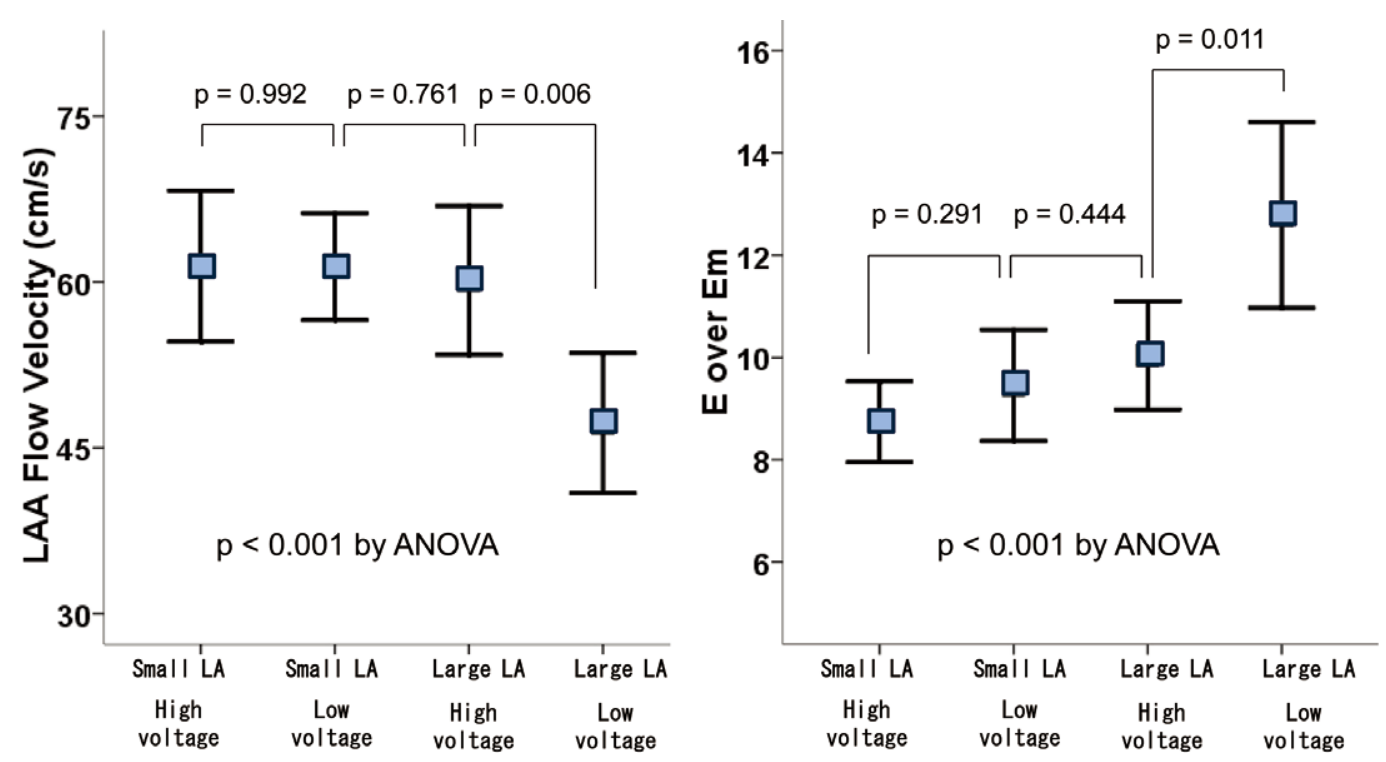

Figure 3. Comparisons of left atrial (LA) mechanical function and left ventricular diastolic function based on atrial size and endocardial voltage. LAA, left atrial appendage. 
forth the argument that LV dysfunction develops in response to remodeling of the fibrillating atria. ${ }^{26,27}$ Our study proved that there exists a correlation between LAA-FV and E/Em in PAF, which was even more significant in older patients with a larger LA, and that the degree of electroanatomical remodeling affects the LAA-FV and E/Em in these patients. Hence, it appears that as LA pressure increases to compensate for impaired LV function and to maintain adequate filling, the overload on the LA first precipitates changes in endocardial voltage, which then evokes changes in the physical structure. $^{6-8}$ Finally, progressive remodeling of the atrium increases the likelihood of developing AF. In this study, we systematically revealed the pathophysiological aspects of AF with comprehensive data that included echocardiography, CT, and electroanatomical voltage mapping. This is in contrast to the previous fragmentary studies that have attempted to prove the relationships of LAA-FV, ${ }^{28} \mathrm{LA}$ remodeling, ${ }^{17,18} \mathrm{LV}$ diastolic function, ${ }^{29}$ and LAA thrombus formation or the events of ischemic stroke. According to our study, among the diverse mechanisms leading to electroanatomical remodeling of the LA in PAF, LV diastolic dysfunction is a principal mechanism, especially in old patients with a large LA. Therefore, careful monitoring of heart rhythm and clinical events is mandatory in patients with PAF and LV diastolic dysfunction, because potentially deranged LA function with high $\mathrm{E} / \mathrm{Em}$ is related to the risk of stroke. In subjects over 55 years of age with an enlarged LA $(\geq 40 \mathrm{~mm})$, LAA-FV reflecting LA mechanical function can be indirectly estimated by E/Em without TEE evaluation, which is a semi-invasive procedure for patients with PAF.

\section{Study Limitations}

This study was a retrospective observational study that included a highly selected group of patients referred for AF catheter ablation. In order for it to comprise only the data from patients whose LAA-FV was measured during sinus rhythm, patients with persistent AF were excluded. Although we found a close relationship to exist among LA function, LA remodeling, and $\mathrm{LV}$ diastolic function, we did not prove the nature of this relationship to be causal. Spontaneous termination of AF has been reported to be associated with stunning of the LA. ${ }^{30,31}$ Therefore, we cannot exclude that LAA stunning affected the LAA-FV. Although the LAA-FV (TEE) and LA voltage (NavX) were measured in sinus rhythm, TTE or cardiac CT was not always done in sinus rhythm. Therefore, there might be some discrepancies between LA function and LA volume depending on rhythm status. Because the endocardial voltage was measured by point-by-point contact mapping, the values used to draw the voltage map may not have accurately represented a spatiotemporally homogeneous distribution of endocardial voltage. The $3 \mathrm{D}$ voltage map analysis was performed with 2D measurements.

\section{Conclusions}

LA mechanical function was closely related to the degree of LA remodeling and LV diastolic function in PAF patients, according to systematic analyses of LAA-FV, TTE parameters, 3D-CT, and electrogram-based voltage mapping. The contribution of LV diastolic dysfunction to LA electroanatomical remodeling was especially significant in older patients with a large LA diameter.

\section{Acknowledgments}

This work was supported by a grant (A085136) from the Korea Health 21 R\&D Project, Ministry of Health and Welfare, and a grant (2010-0010537) from the Basic Science Research Program of the National Research Foundation of Korea under the Ministry of Education, Science and Technology of the Republic of Korea.

\section{Disclosure}

No conflict of interest declared.

\section{References}

1. Tan AY, Zimetbaum P. Atrial fibrillation and atrial fibrosis. J Cardiovasc Pharmacol 2011; 57: 625-629.

2. Kopecky SL, Gersh BJ, McGoon MD, Whisnant JP, Holmes DR Jr, Ilstrup DM, et al. The natural history of lone atrial fibrillation: A population-based study over three decades. N Engl J Med 1987; 317: 669-674.

3. Wijffels MC, Kirchhof CJ, Dorland R, Allessie MA. Atrial fibrillation begets atrial fibrillation: A study in awake chronically instrumented goats. Circulation 1995; 92: 1954-1968.

4. Benjamin EJ, D'Agostino RB, Belanger AJ, Wolf PA, Levy D. Left atrial size and the risk of stroke and death: The Framingham Heart Study. Circulation 1995; 92: 835-841.

5. Chugh SS, Blackshear JL, Shen WK, Hammill SC, Gersh BJ. Epidemiology and natural history of atrial fibrillation: Clinical implications. J Am Coll Cardiol 2001; 37: 371-378.

6. Tsang TS, Barnes ME, Gersh BJ, Bailey KR, Seward JB. Left atrial volume as a morphophysiologic expression of left ventricular diastolic dysfunction and relation to cardiovascular risk burden. Am J Cardiol 2002; 90: 1284-1289.

7. Tsang TS, Gersh BJ, Appleton CP, Tajik AJ, Barnes ME, Bailey KR, et al. Left ventricular diastolic dysfunction as a predictor of the first diagnosed nonvalvular atrial fibrillation in 840 elderly men and women. J Am Coll Cardiol 2002; 40: 1636-1644.

8. Pritchett AM, Mahoney DW, Jacobsen SJ, Rodeheffer RJ, Karon BL, Redfield MM. Diastolic dysfunction and left atrial volume: A population-based study. J Am Coll Cardiol 2005; 45: 87-92.

9. Kurt M, Wang J, Torre-Amione G, Nagueh SF. Left atrial function in diastolic heart failure. Circ Cardiovasc Imaging 2009; 2: 10-15.

10. Lang RM, Bierig M, Devereux RB, Flachskampf FA, Foster E, Pellikka PA, et al. Recommendations for chamber quantification: A report from the American Society of Echocardiography's Guidelines and Standards Committee and the Chamber Quantification Writing Group, developed in conjunction with the European Association of Echocardiography, a branch of the European Society of Cardiology. $J$ Am Soc Echocardiogr 2005; 18: 1440-1463.

11. Helak JW, Reichek N. Quantitation of human left ventricular mass and volume by two-dimensional echocardiography: In vitro anatomic validation. Circulation 1981; 63: 1398-1407.

12. Park JH, Pak HN, Choi EJ, Jang JK, Kim SK, Choi DH, et al. The relationship between endocardial voltage and regional volume in electroanatomical remodeled left atria in patients with atrial fibrillation: Comparison of three-dimensional computed tomographic images and voltage mapping. J Cardiovasc Electrophysiol 2009; 20: $1349-$ 1356.

13. Pollick C, Taylor D. Assessment of left atrial appendage function by transesophageal echocardiography: Implications for the development of thrombus. Circulation 1991; 84: 223-231.

14. Fatkin D, Kelly RP, Feneley MP. Relations between left atrial appendage blood flow velocity, spontaneous echocardiographic contrast and thromboembolic risk in vivo. J Am Coll Cardiol 1994; 23: 961-969.

15. Zabalgoitia M, Halperin JL, Pearce LA, Blackshear JL, Asinger RW, Hart RG. Transesophageal echocardiographic correlates of clinical risk of thromboembolism in nonvalvular atrial fibrillation: Stroke Prevention in Atrial Fibrillation III Investigators. J Am Coll Cardiol 1998; 31: 1622-1626.

16. Maehama T, Okura H, Imai K, Yamada R, Obase K, Saito K, et al. Usefulness of CHADS2 score to predict C-reactive protein, left atrial blood stasis, and prognosis in patients with nonrheumatic atrial fibrillation. Am J Cardiol 2010; 106: 535-538.

17. Park JH, Joung B, Son NH, Shim JM, Lee MH, Hwang C, et al. The electroanatomical remodelling of the left atrium is related to CHADS/ CHADSVASc score and events of stroke in patients with atrial fibrillation. Europace 2011; 13: 1541-1549.

18. Chao TF, Cheng CC, Lin WS, Tsao HM, Lin YJ, Chang SL, et al. Associations among the CHADS(2) score, atrial substrate properties, and outcome of catheter ablation in patients with paroxysmal atrial fibrillation. Heart Rhythm 2011; 8: 1155-1159.

19. Moon J, Hong YJ, Shim J, Hwang HJ, Kim JY, Pak HN, et al. Right atrial anatomical remodeling affects early outcomes of nonvalvular atrial fibrillation after radiofrequency ablation. Circ J 2012; 76: 860- 
867.

20. Kim SK, Park JH, Kim JY, Choi JI, Joung B, Lee MH, et al. High plasma concentrations of transforming growth factor-beta and tissue inhibitor of metalloproteinase-1: Potential non-invasive predictors for electroanatomical remodeling of atrium in patients with nonvalvular atrial fibrillation. Circ J 2011; 75: 557-564.

21. Verma A, Wazni OM, Marrouche NF, Martin DO, Kilicaslan F, Minor S, et al. Pre-existent left atrial scarring in patients undergoing pulmonary vein antrum isolation: An independent predictor of procedural failure. J Am Coll Cardiol 2005; 45: 285-292.

22. Rosca M, Lancellotti P, Popescu BA, Pierard LA. Left atrial function: Pathophysiology, echocardiographic assessment, and clinical applications. Heart 2011; 97: 1982-1989.

23. Hu YF, Hsu TL, Yu WC, Huang SH, Tsao HM, Tai CT, et al. The impact of diastolic dysfunction on the atrial substrate properties and outcome of catheter ablation in patients with paroxysmal atrial fibrillation. Circ J 2010; 74: 2074-2078.

24. Jais P, Peng JT, Shah DC, Garrigue S, Hocini M, Yamane T, et al. Left ventricular diastolic dysfunction in patients with so-called lone atrial fibrillation. J Cardiovasc Electrophysiol 2000; 11: 623-625.

25. Agmon Y, Khandheria BK, Meissner I, Petterson TM, O'Fallon WM, Wiebers DO, et al. Are left atrial appendage flow velocities adequate surrogates of global left atrial function? A population-based transthoracic and transesophageal echocardiographic study. J Am Soc Echocardiogr 2002; 15: 433-440.

26. Reant P, Lafitte S, Jais P, Serri K, Weerasooriya R, Hocini M, et al.
Reverse remodeling of the left cardiac chambers after catheter ablation after 1 year in a series of patients with isolated atrial fibrillation. Circulation 2005; 112: 2896-2903.

27. Therkelsen SK, Groenning BA, Svendsen JH, Jensen GB. Atrial and ventricular volume and function in persistent and permanent atrial fibrillation, a magnetic resonance imaging study. J Cardiovasc Magnetic Res 2005; 7: 465-473.

28. Iwakura K, Okamura A, Koyama Y, Date M, Higuchi Y, Inoue K, et al. Effect of elevated left ventricular diastolic filling pressure on the frequency of left atrial appendage thrombus in patients with nonvalvular atrial fibrillation. Am J Cardiol 2011; 107: 417-422.

29. Taguchi Y, Takashima S, Hirai T, Fukuda N, Ohara K, Nakagawa K, et al. Significant impairment of left atrial function in patients with cardioembolic stroke caused by paroxysmal atrial fibrillation. Intern Med 2010; 49: 1727-1732.

30. Louie EK, Liu D, Reynertson SI, Loeb HS, McKiernan TL, Scanlon $\mathrm{PJ}$, et al. "Stunning" of the left atrium after spontaneous conversion of atrial fibrillation to sinus rhythm: Demonstration by transesophageal Doppler techniques in a canine model. J Am Coll Cardiol 1998; 32: $2081-2086$

31. Grimm RA, Stewart WJ, Arheart K, Thomas JD, Klein AL. Left atrial appendage "stunning" after electrical cardioversion of atrial flutter: An attenuated response compared with atrial fibrillation as the mechanism for lower susceptibility to thromboembolic events. J Am Coll Cardiol 1997; 29: 582-589. 